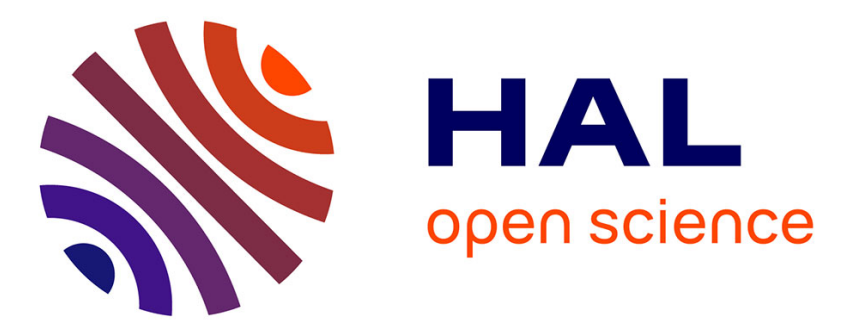

\title{
Evaluating the uncertainty for a complex experiment:the case of the plasma potential measurement
}

\author{
Valentin Pigeon, Magali Muraglia, Cécile Arnas, Nicolas Claire, Lenaic \\ Couëdel
}

\section{- To cite this version:}

Valentin Pigeon, Magali Muraglia, Cécile Arnas, Nicolas Claire, Lenaic Couëdel. Evaluating the uncertainty for a complex experiment:the case of the plasma potential measurement. European Journal of Physics, 2020, 41 (3), pp.035806. 10.1088/1361-6404/ab7830 . hal-02566174

\section{HAL Id: hal-02566174 \\ https://hal.science/hal-02566174}

Submitted on 14 May 2020

HAL is a multi-disciplinary open access archive for the deposit and dissemination of scientific research documents, whether they are published or not. The documents may come from teaching and research institutions in France or abroad, or from public or private research centers.
L'archive ouverte pluridisciplinaire HAL, est destinée au dépôt et à la diffusion de documents scientifiques de niveau recherche, publiés ou non, émanant des établissements d'enseignement et de recherche français ou étrangers, des laboratoires publics ou privés. 
ACCEPTED MANUSCRIPT

\section{Evaluating the uncertainty for a complex experiment:the case of the plasma potential measurement}

To cite this article before publication: Valentin Pigeon et al 2020 Eur. J. Phys. in press https://doi.org/10.1088/1361-6404/ab7830

\section{Manuscript version: Accepted Manuscript}

Accepted Manuscript is "the version of the article accepted for publication including all changes made as a result of the peer review process, and which may also include the addition to the article by IOP Publishing of a header, an article ID, a cover sheet and/or an 'Accepted

Manuscript' watermark, but excluding any other editing, typesetting or other changes made by IOP Publishing and/or its licensors"

This Accepted Manuscript is (C) 2020 European Physical Society.

During the embargo period (the 12 month period from the publication of the Version of Record of this article), the Accepted Manuscript is fully protected by copyright and cannot be reused or reposted elsewhere.

As the Version of Record of this article is going to be / has been published on a subscription basis, this Accepted Manuscript is available for reuse under a CC BY-NC-ND 3.0 licence after the 12 month embargo period.

After the embargo period, everyone is permitted to use copy and redistribute this article for non-commercial purposes only, provided that they adhere to all the terms of the licence https://creativecommons.org/licences/by-nc-nd/3.0

Although reasonable endeavours have been taken to obtain all necessary permissions from third parties to include their copyrighted content within this article, their full citation and copyright line may not be present in this Accepted Manuscript version. Before using any content from this article, please refer to the Version of Record on IOPscience once published for full citation and copyright details, as permissions will likely be required. All third party content is fully copyright protected, unless specifically stated otherwise in the figure caption in the Version of Record.

View the article online for updates and enhancements. 


\title{
Evaluating the uncertainty for a complex experiment: the case of the plasma potential measurement
}

\author{
V. Pigeon ${ }^{1}$, M. Muraglia ${ }^{1,2}$, C. Arnas $^{1}$, N. Claire $^{1}$, L. Couëdel ${ }^{1,3}$
}

${ }^{1}$ Aix-Marseille Université, CNRS, PIIM UMR 7345, Marseille, France

2 Aix-Marseille Université, France-Japan Laboratory, LIA, Marseille, France

${ }^{3}$ Physics and Engineering Physics, University of Saskatchewan, Saskatoon, Saskatchewan, Canada

E-mail: valentin.pigeon@univ-amu.fr

\section{Abstract.}

A general method for the determination of the uncertainty of a physical quantity measured during an experiment is presented. This procedure is described step-by-step so it can be applied to any measurement and experiment available for undergraduate and graduate students, from the simplest to the most complex. The method is then applied to a classic measurement of plasma physics: the plasma potential determination using an emissive probe. This kind of experiments is routinely performed in plasma physics laboratories and may be realized by graduate students. The emissive probe diagnostic relies on a data analysis method called the inflection point. This method follows an indirect procedure on which the step-by-step uncertainty calculation strategy presented in the first part of the article is presented. 
Evaluating the uncertainty for a complex experiment: the case of the plasma potential measurement2

\section{Introduction}

In laboratory experiments, the measurement of physical quantities is necessary to characterize and understand the studied phenomena, and to validate theoretical models and numerical simulations. However, it is crucial to characterize properly the measurement in order to obtain meaningful informations. Comparing a measured physical quantity and its theoretical value leads to the following statement: in theoretical models, physical quantities are implicitly given by numerical values with an infinite precision (i.e. exempted from errors). However, this statement is wrong for the measured physical quantities. Indeed, two consecutive measurements of the same physical quantity under the same experimental conditions (i.e. keeping the same repeatable conditions [1] $\ddagger$ ), give two different results because of measurement uncertainty. This notion of uncertainty is essential in metrology. The mathematical nature of a measurement result does not consist of one single value exempted from errors as in theoretical models but rather of a random variable $Y$. One has to determine a "true value" $\bar{y}$ [1], which can be interpreted as the value of $Y$ with the highest probability of measurement, and an uncertainty on the measurement $u$ which takes into account the different sources of uncertainties [2].

Let us consider that an infinite number of $Y$ measurements under the same experimental conditions can be performed. According to the Central Limit Theorem (LCT) [3], the measurement result can be described by a continuum random variable whose probability density $f(y)$ is given by a normal law, centered around a theoretical average value $\mu$ and a theoretical standard deviation $\sigma$ (see Fig.1). $\mu$ and $\sigma$ are theoretical for two reasons. Firstly, it is not possible to keep exactly the same experimental conditions for each measurement, which leads to variations of every parameter including the measured quantity. Secondly, it is obviously impossible to proceed to an infinite number of measurements. The first statement leads to "systematic errors"[1] which systematically shift $\mu$ to $\mu_{1}$ and contribute to the uncertainty of the measurement modifying $\sigma$ to $\sigma_{1}$ (see Fig.1). The second statement leads to the "random error" [1] which originates from the sampling of $Y$. From $n$ measurements of $Y$ one cannot deduce $\mu_{1}$ and $\sigma_{1}$ but rather estimations of these two quantities (see Fig.1). Consequently, to have a meaningful experimental value of $Y, \bar{y}$ and $u$ - which takes into account both the random and systematic errors of the measurement - must be provided.

For each experimental measurement of a physical quantity, it is always possible to rigorously determine its uncertainty based on the analysis of the measurement process. In the metrology science literature, numerous metrological procedures are available for the measurements of temperature, mass, resistance... However as soon as the measurement gets more complex, finding a metrological method providing the uncertainty becomes tougher. Often, the measurement uncertainty consists of the calculation of a mean and a standard deviation at worst ; a systematic error

\$ In the following, Ref [1] is used to reference words that are defined in the international vocabulary of metrology. 
Evaluating the uncertainty for a complex experiment: the case of the plasma potential measurement3

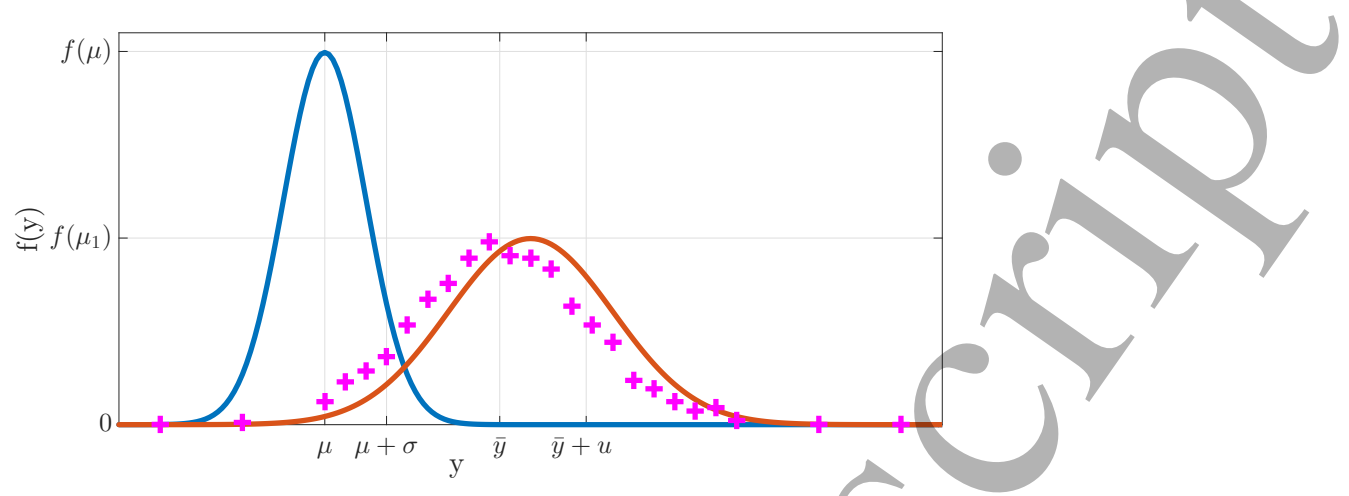

Figure 1. Blue line - Distribution function of the measurand $Y$ for an infinite number of measurements in the same conditions ( $i$. e. without systematic errors) associated to the statistical characteristics $\mu$ and $\sigma$. Red line - Distribution function of the measurand $Y$ for an infinite number of measurements with systematic errors associated to the statistical characteristics $\mu_{1}$ and $\sigma_{1}$. Pink crosses - Distribution function of the $Y$ measurand for $\mathrm{n}$ measurements associated to the statistical characteristics $\bar{y}$ and $u$ calculating from the sampling.

related to the measuring instrument is added at best. Unfortunately, the presentation of scientific results from laboratory experiments (carried out by students or even scientists) is presented too often with an uncertainty that is not correctly calculated (the measurement uncertainty method is often not presented). However, a metrological analysis is necessary to correctly determine the average value (which can be considered as the true value of the measurement result) and its associated uncertainty. In addition, a rigorous metrological analysis of the measurement chain also allows the students/scientists to have a better understanding of the results of the experiments. It is common to think that out of academic cases (metrology of mass, temperature, length measurement for instance) performing a rigorous metrological study is not possible. However it as actually achievable. The purpose of the present article is to invite physics students (undergraduate and graduate students) to think about the concept of experimental measurements in terms of random variables and probabilities. A good understanding of measurement concepts is essential to appreciate the importance of uncertainties and to learn how to correctly evaluate them.

This issue occurs is all field of physics. In the present article we will use plasma physics as an example. One of the simplest plasma physics experiments (available for graduate students) is the plasma potential determination using an emissive probe. The measurement procedure considered here is called the inflection point. When performing this experiments, it is here again mandatory to present the results with the proper uncertainty. However, detailed examples are missing in the literature. Thus, the purpose of this paper is to provide a metrological procedure usable for any measurements independentely of the complexity. In particular, this work intends to give a clear and pedagogical procedure to determine the uncertainty on the plasma potential measurement using an emissive probe [7]. Indeed, with density and temperature, plasma 


\section{Evaluating the uncertainty for a complex experiment: the case of the plasma potential measurement4}

potential is one of the most important plasma parameters. Its measurement is necessary to understand a wide range of plasma phenomena. For this purpose, emissive probes are used in numerous plasma devices [8, 10, 9], and the theoretical modelling of these probes is well developed $[11,10,12,13,14]$. Emissive probes are said to be one of the best diagnostic to measure the plasma potential [5], and several methods have been proposed for the plasma potential measurement $[7,10,15,16]$. Howeyer, the uncertainty of such measurement has never been carefully analyzed. We propose in this work a rigorous uncertainty evaluation of the plasma potential measurement using emissive probe in a unperturbed plasma. This is an ideal example: most often the error of such a measurement is calculated simply from several repeated measurements, the systematic errors being omitted. The determination of the plasma potential consists of the measurement of a current and a voltage. Therefore, an extended knowledge in plasma physics is not required and the measurement chain and procedure can be analyzed as it is.

The paper is organized as follow. After the introduction, Section II presents the statistics underlying the measurement of a physical quantity. More precisely, the determination of the uncertainty of a measurement from the random error and the systematic errors is presented step-by-step. In section III, the experimental apparatus and the measuring chain, used for the measurement of the plasma potential $\phi_{p}$ by an emissive probe, are briefly described. In section IV, the step-by-step procedure of the uncertainty determination described in section II is applied to the measurement of the potential plasma with an emissive probe. A pedagogical procedure is given to proceed to the uncertainty calculation. In the section $\mathrm{V}$, experimental results are presented and discussed. Finally, in the section VI, the main conclusions of this work are presented.

\section{Statistical description of a measurement result : the measurand is a random variable}

According to Ref. [2], a measurement [1] is an experimental process that aims to obtain one or more values that can reasonably be attributed to a measurand $Y$, which is the quantity to be measured. However, attempting to get a "true value" is not possible since $Y$ is by nature a random variable: its value is undertermined. From a theoretical point of view, $Y$ is a continuous quantity generally defined on $\mathbb{R}$. Consequently, the aim of a measurement is to determine a "mean" value of $Y, \bar{y}$, which is called the conventional true value of $Y$ and the uncertainty of the measurement $u$. $u$ represents the dispersion around $\bar{y}$ of the different measured value of $Y$ with a reasonable probability. (For example, if one wants to measure the temperature of a classroom, the probability to measure $-100^{\circ} \mathrm{C}$ is close to 0 while the probability to measure $25^{\circ} \mathrm{C}$ is close to the maximum probability.) Thus, the result of a measurement can be given as $Y=\bar{y} \pm u$ (it will be shown later that usually the result is rather given as $Y=\bar{y} \pm I$ where $I$ is the expanded measurement uncertainty [1]).

A measurement is always marred by errors. These errors have to be determined to 
Evaluating the uncertainty for a complex experiment: the case of the plasma potential measurement5

establish the uncertainty of the measurement. Two kinds of errors exist : systematic errors and random error.

\subsection{Systematic errors}

Systematic errors are defined as the component of the measurement errors that remains constant or varies in a predicable way during repeated measurements. For a given measurement, systematic errors are obtained from a detailed and rational analysis [17] of the measurement procedure. Usually, it is convenient to organize the different systematic errors in a "5-M diagram" (see Fig.2). Once systematic errors are listed, a model for the measurement process has to be derived, accounting for the systematic errors $x_{1}, x_{2}$, $\ldots x_{m} \S$ :

$$
Y=f\left(\bar{y}, x_{1}, x_{2}, \ldots, x_{m}\right)
$$

From the uncertainty propagation law comes the combined standard measurement uncertainty due to systematic errors [18]:

$$
u^{\text {sys }}=\sqrt{\sum_{i=1}^{m}\left(\frac{\partial f}{\partial x_{i}}\right)_{x_{i}=\bar{x}_{i}}^{2}\left(u_{i}^{s y s}\right)^{2}}
$$

where $\bar{x}_{i}$ is a mean value of $x_{i}$ and $u_{i}^{s y s}$ is the standard measurement uncertainty of the systematic error labelled $i$ which have to be determined. It may be noted that Eq.2 is derived assuming the independence of the variables $x_{i}$. Eq.2 arises from a second order Taylor expansion and assumes that systematic errors are small. A derivation of the uncertainty propagation law in the case, in of correlated variables can be found in [18].

To compute $u^{\text {sys }}$ from Eq.2, the standard measurement uncertainties $u_{i}^{\text {sys }}$ has to be determined. From the definition of a systematic error, it is impossible to extract the uncertainty of a systematic error by statistical calculations : one cannot proceed to repeated observations to evaluate a systematic error. As an example, the balance needle involves a systematic error which is constant from one measurement to another. As a consequence, the standard deviation of that shift is 0 . Thus, to determine the standard uncertainty $u_{i}^{\text {sys }}$ of a systematic error $x_{i}$, one should analyze the origin of the error from available informations (experience, knowledge, previous experimental data, manufacturer's specifications, ...) on the possible variability of $x_{i}$. The method to determine standard uncertainty of systematic error is called in the literature "type B evaluation" [1, 2]. Some examples are:

- The measuring instrument induces a systematic error on the measurement linked to its metrological properties (i.e. instrumental bias, instrumental drift, instrumental $\S$ Influence quantities [1] (for example, temperature in shim length measurement) and indirect quantities (for indirect measurement such as electric resistance in a voltage measurement) involve systematic errors which are taken into account in the measurement model (Eq.1). Moreover, if a systematic error is well known, it can be directly corrected. In this case, the correction induces a systematic error and is an input quantity in the measurement model. 


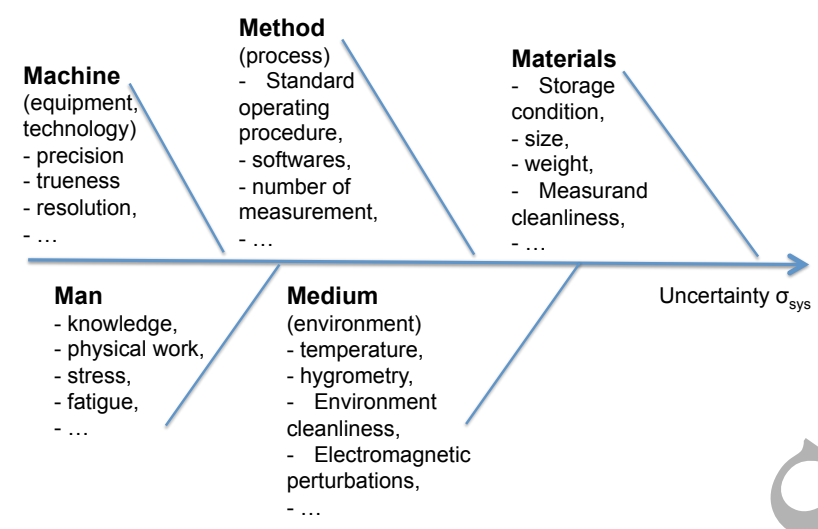

Figure 2. The "5-M diagram" or the "Ishikawa diagram"[17] provides a detailed and rational analysis of the measurement process to determine systematic errors.

precision). The standard uncertainty is obtained from the instrument specifications. If the instrument has been calibrated, the calibrated uncertainty of the instrument $I_{c a l}$ extended by a coverage factor $k$ are available. Thus, the standard uncertainty due to the calibrated measuring instrument is given by $u_{c a l}^{\text {sys }}=I_{c a l} / k$.

- If the instrument is not calibrated, the specifications notes give the accuracy class of the instrument. This class provides indications on the metrological properties and on the level of confidence of the instrument. More precisely, the class indicates the uncertainty interval of the measure given by the instrument. The normal law is usually applied to the interval to determine the standard systematic uncertainty on the measurement $u_{i n s t}^{\text {sys }}$. Indeed, the/value given by an instrument is also a random variable which is the result of a lot of independent factors allowing the use of the LCT.

- The resolution $q$ of the instrument display adds a systematic error to the measurement. Indeed, for a displayed value $y$ of the measurand, all the values $[y-q / 2, y+q / 2]$ could be measured with the same probability. As a consequence, the standard uncertainty associated to the display resolution can be calculated using an uniform probability law which represents equiprobable experiences.

More generally, if the physical phenomenon inducing the systematic error $x_{i}$ is in the measuring interval $\left[y-y^{-}, y+y^{+}\right]$, it should be carefully analyzed in order to find a theoretical statistical distribution function $f$ which is appropriate to determine the standard systematic uncertainty $u_{i}^{\text {sys }}$ from the statistical formula :

$$
u_{i}^{\text {sys }}=\sqrt{\int_{y^{-}}^{y^{+}}\left[y-\left(\int_{y^{-}}^{y^{+}} y f(y) d y\right)\right]^{2} f(y) d y}
$$

The nature of the phenomenon at the origin of the systematic error determines $f$. If various independent factors are implied, the LCT can be applied and $f$ follows a normal law. If all the values of $\left[y-y^{-}, y+y^{+}\right]$have the same probability, $f$ follows an uniform 
1

2

3

4

5

6

7

8

9

Evaluating the uncertainty for a complex experiment: the case of the plasma potential measurement7 law. If the measurand is periodic on the interval $\left[y-y^{-}, y+y^{+}\right], f$ follows an arcsinus law.

\subsection{Random error}

Together with systematic errors exists the random error. The standard definition of the random error can be found in [1]. From a theoretical point of view, an infinite number of the measurand measurements in the same conditions allows the use of the LCT, which gives the value of the theoretical mean value $\mu_{1}$ and of the standard deviation $\sigma_{1}$ associated to the normal law while taking into account the systematic errors (See Fig.1-a). There is then no random error and the associated standard measurement uncertainty $u^{\text {rand }}$ is equal to 0 . However, it is obviously impossible to proceed to an infinite number of measurements and by nature $\mu_{1}$ and $\sigma_{1}$ are unknown and undermined (as well as $\mu$ and $\sigma$ on Fig.1-a). Only a finite number $n$ of measurements (Fig.1-c) can be performed, leading the random error. The random error is the error obtained from $\mathrm{n}$ measurements made in identical conditions. The $n$ measurement value $\left[y_{1}, y_{2}, \ldots, y_{n}\right]$ fluctuates around a mean value

$$
\bar{y}=\sum_{i=1}^{n} y_{i} / n
$$

with a standard deviation $\sqrt{\sum_{i=1}^{n} \frac{\left(y_{i}-\bar{y}\right)^{2}}{n}}$. As a consequence, to determine the statistical standard deviation $u^{\text {rand }}$, which represents the contribution of the random error on the uncertainty measurement $u, \mu_{1}$ and $\sigma_{1}$ have to be estimated from the sampling of $n$ independent random variables $y_{1}, y_{2}, \ldots y_{n}$. Following Ref [3], it can be shown that a good estimation of $\mu_{1}$ is $\bar{y}$ and a good estimation of $\sigma_{1}$ is $\sigma_{1}^{\text {est }}=$ $\sqrt{\sum_{i=1}^{n} \frac{\left(y_{i}-\bar{y}\right)^{2}}{n-1}}(\bar{y}$ is not the "true" mean value of the sampling, but an estimation as any other $y_{i}$ ). Usually, $\bar{y}$ is taken as the conventional true quantity value [1] of the measurand. By definition, the random contribution to the uncertainty measurement $u^{\text {rand }}$ is the standard deviation of the random variable $\bar{y}$ and according to [3], it can be shown that if $n$ is large enough $\|$ the LCT can be applied

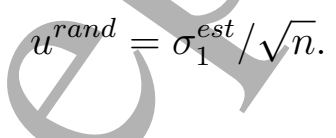

\subsection{Measurement uncertainty evaluation procedure}

To summarize, the following steps are necessary to a proper evaluation of the uncertainty $u$ associated to the measurement result of a measurand $y$ :

$\|$ To know the measurement number $n$ required to applied the LCT, one should plot $u^{\text {rand }}$ as a function of $n$. The "good" value of $n$ is its the minimal value for which an increase does not lead to a noticeably decrease of $u^{\text {rand }}$. The random error varies like $1 / n^{2}$, usually $n \sim 30$. 


\section{(i) Measurement modeling 9}

To determine the uncertainty associated to a measurement, its mathematical model has to be derived :

$$
y=f\left(z_{1}, z_{2}, \ldots, z_{p}\right) \quad,
$$

where $z_{j}$ represents measured physical quantities (or physical constants known with their uncertainty). For simplicity, the quantities $z_{j}$ are supposed independent of each other ${ }^{+}$(for correlated quantities see $[1,3]$ ). At this step, the propagation law can be applied to get the expression of $u$ :

$$
u=\sqrt{\sum_{j=1}^{p}\left(\frac{\partial f}{\partial z_{j}}\right)^{2}\left(u_{z_{j}}\right)^{2}} .
$$

$u_{z_{j}}$ is the uncertainty of $z_{j}$ and should be determined. If $z_{j}$ is not measured and represents a physical constant, its uncertainty $u_{z_{j}}$ is known (by manufacturer's documents, equal to 0 for physical fundamental constant, ...). When $z_{j}$ is a measured quantity, $u_{z_{j}}$ can be determined following the steps described below and has two components : one derived from the random error treatment and one derived from the systematic errors treatment.

(ii) Random error treatment*

Proceed to $n$ measurements of each measured quantity $z_{j}:\left\{z_{j_{1}}, z_{j_{2}} \ldots z_{j_{n}}\right\}$. The conventional true value $\bar{z}_{j}$ and the random contribution to the measurement uncertainty $u_{z_{j}}^{\text {rand }}$ can be obtained for the measurand $z_{j}$ using Eqs.4-5 :

$$
\bar{z}_{j}=\sum_{i=1}^{n} \frac{z_{j_{i}}}{n}, \quad u_{z_{j}}^{\text {rand }}=\sqrt{\sum_{i=1}^{n} \frac{\left(z_{j_{i}}-\bar{z}_{j}\right)^{2}}{n(n-1)}} .
$$

It may be noted that $n$ should be large enough in order to satisfy the application conditions of the LCT.

\section{(iii) Systematic errors treatment}

For each measured quantity $z_{j}$, the measurement process has to be analyzed in details ( $5 \mathrm{M}$-method) to determine the sources of systematic errors $x_{1}, x_{2}, \ldots, x_{m}$.

I As an exemple, the measurement of a electric current intensity $\mathcal{I}$ passing through a resistor $\mathcal{R}$ from the voltage measurement $\mathcal{U}$ across the resistor. The mathematic model is given by the well know Ohm's law, $\mathcal{I}=\mathcal{U} / \mathcal{R} . \mathcal{I}$ is indirectly measured by the measurement of $\mathcal{U}$ and knowing $\mathcal{R}$. Applying the propagation laww, the uncertainty of the indirect measurement of $\mathcal{I}$ is $u=\sqrt{\mathcal{R}^{2} * u_{\mathcal{U}}^{2}+\mathcal{U}^{2} * u_{\mathcal{R}}^{2}}$. Here, the value of $\mathcal{U}$ could be a mean value obtained from $n$ measurements. $\mathcal{R}$ is known and its uncertainty $u_{\mathcal{R}}$ should be find in the constructor's document provided with the resistor. It remains to be determined $u_{\mathcal{U}}$ from a random error treatment ( $n$ measurements of $\mathcal{U}$ - step $2, u_{z_{j}}^{\text {rand }}$ ) and a systematic errors treatment of the $\mathcal{U}$ measure process (step 3 ).

+ One can note that if the measurand is directly measured the model is very simple, $y=y$ and $u$ can be determined thanks to a random error treatment (step 2) and a systematic errors treatment (step 3).

In the example of the Ohm's law, the only quantity directly measured is $\mathcal{U}$. To determine the contribution of the random error to $\mathcal{u}_{\mathcal{U}}$, one has to proceed to $n$ measurements of $\mathcal{U},\left\{\mathcal{U}_{1}, \mathcal{U}_{2}, \ldots \mathcal{U}_{n}\right\}$. From Eqs.4-5, one can calculate $\overline{\mathcal{U}}=\sum_{j=1}^{n} \frac{\mathcal{U}_{j}}{n}$ and $u_{\mathcal{U}}^{\text {rand }}=\sqrt{\sum_{j=1}^{n} \frac{\left(\mathcal{U}_{j}-\overline{\mathcal{U}}\right)^{2}}{n(n-1)}}$ 


\section{Plasma potential measurement of a gas discharge using an emissive probe}

The metrological procedure exposed in the last section can be applied to any physical quantity measured by any method to correctly determine the measurement uncertainty. As an example, we present in the following the metrological analysis of the plasma potential measurement in a gas discharge using an emissive probe. The determination of the potential implies the measurement of the probe voltage and corresponding current. Usually, for such experiments, the uncertainty is determined only by calculating the standard deviation of a limited number of measures, $i$. e. taking into account only the random error and neglecting the systematic errors which are thought not easy or impossible to evaluate. However, using the different steps presented in the previous section, one can determine the systematics errors and their associated uncertainty.

The plasma state is one of the four fundamental states of matter and was first described by I. Langmuir [4] in the 1920s. It is an ionized gas composed of ions, electrons and atoms (the neutral gas) free to move in all directions of space, and the interactions between the charged particles are governed by the Coulomb interaction. Thus a plasma is a medium with a collective behavior and is neutral at macroscopic scales. The number of plasma applications is large: controlled thermonuclear fusion, astrophysics (Univers is composed of $99 \%$ of plasma), plasma chemistry (formation of 


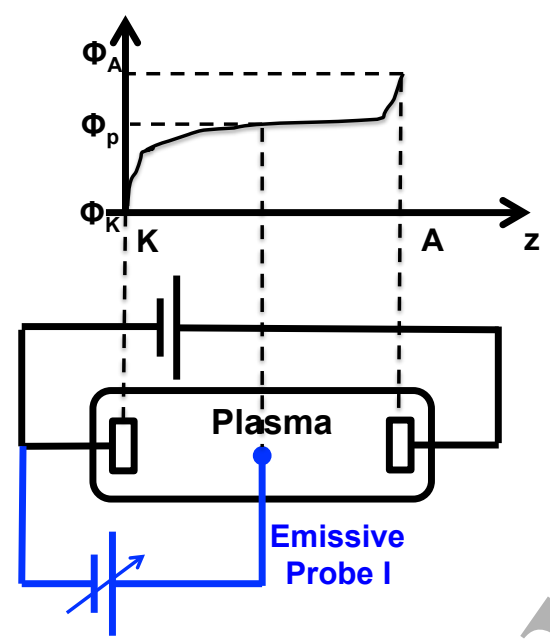

Figure 3. Example of a plasma discharge experimental apparatus. An emissive probe is placed in the plasma center. The current $I$ on the probe is measured to determine the plasma I-V trace.

Ozone from $\mathrm{O}_{2}$, dissociation of $\mathrm{CO}_{2}$, catalysis of chemical reactions), industry (surface processing, sterilization of medical items, analytical chemistry, plasma screen, lighting, ion sources...)...

A plasma can be formed by biasing two electrodes - a cathode (K) and an anode (A) - embedded in a low pressure gas (Argon or Neon for instance). The cathode is negatively biased compared to the anode such as $\phi_{K}-\phi_{A}<0$, as shown in Figure 3. Over a threshold voltage the gas is ionized and a current is driven between the electrodes. This is a basic plasma discharge. In plasma physics laboratories, many experiments rely on such discharges and plasma properties have to be measured. Here, we focus on the electric potential (with respect to the ground) at the center of a discharge (Fig.3): the plasma potential $\phi_{p}$, measured using an emissive probe.

\subsection{Emissive probe measurement principle - Inflection point method}

An emissive probe (Figure 4), consisting of a Tungsten hairpin-shaped wire (in order to resist to high temperature), is immersed in the plasma discharge (Fig.3). A current is driven through it in order to heat the wire up to electron emission (the thermionic emission, around $T_{w} \sim 1500-2000^{\circ} \mathrm{C}$ ). One can determine the plasma potential $\phi_{p}$ at the plasma center from the current-voltage I-V characteristic of the probe. It is obtained by biasing the probe with respect to the ground. At voltages below $\phi_{p}$ the probe emits electrons; at voltage over $\phi_{p}$ the probe collects electrons. In Fig.5, a typical $\mathrm{I}-\mathrm{V}$ characteristic is shown: the current measured on the probe is plotted as a function of the probe bias from A to D. The current measured on the probe is a combination of a collected current and an emitted current. As a consequence, the characteristic can be decomposed into 3 regions $(\mathrm{AB}, \mathrm{BC}, \mathrm{CD})$ which correspond the 3 different configurations 


\section{Evaluating the uncertainty for a complex experiment: the case of the plasma potential measurement11}

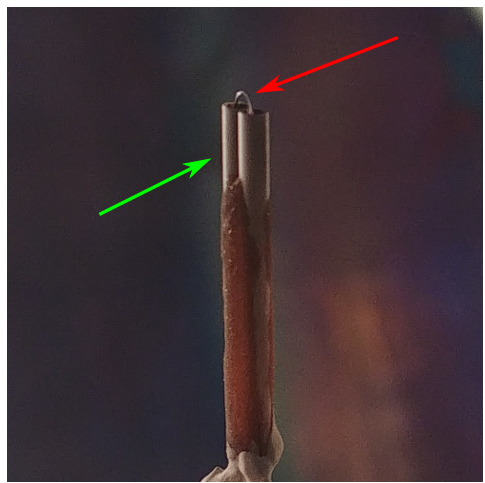

Figure 4. Picture of the emissive probe. The red arrow indicates the hairpin-shaped Tungsten wire, which size is typically 1 to $2 \mathrm{~mm}$. The green arrow shows the ceramic tubes that hold and shield the connection wire.

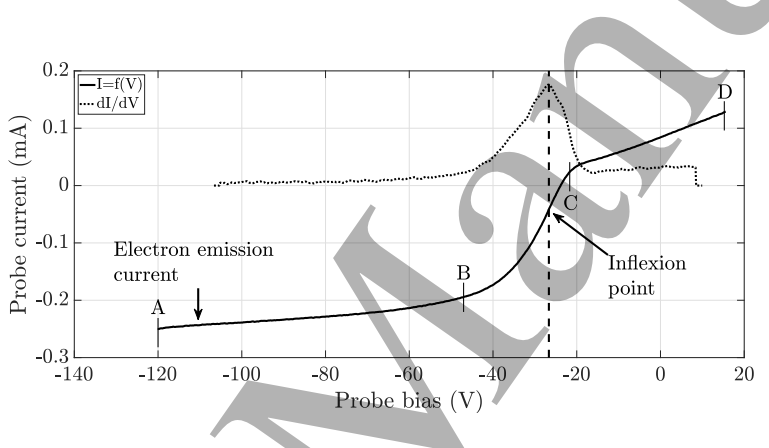

Figure 5. Typical emissive probe $\mathrm{I}-\mathrm{V}$ characteristics, with its first derivative. The inflexion point is simply given by the maximum of the first derivative. The emission current is the mean electronic current at low biases (far enough from the IV-knee).

of the electrostatic sheath surrounding the probe (Fig.5).

The region $\mathrm{AB}$ is characterized by $V<<\phi_{p}$, where the probe collects ions and emits electrons if its temperature is high enough. The resulting current on the probe is given by the addition of the probe emitted current $I_{e 0}$ and the ion saturation current $I_{i}^{\text {sat }}$. Usually the ion saturation current is negligeable $\left(I_{e 0}>>I_{i}^{\text {sat }}\right)$ and $I_{e 0}$ is given by the Richardson-Dushman equation which is $I_{e 0}=A T_{w}^{2} S \exp \left(\frac{e \phi_{w}}{T_{w}}\right)$ [5], where $A$ is the Ridchardson's constant, $\phi_{w}$ is the work function of the wire, $\mathrm{S}$ is the wire surface area and $T_{w}$ the probe temperature.

From $V>>\phi_{p}$ (the region $\mathrm{CD}$ ), the space-charge region surrounding the probe becomes electronic : all the ions are repelled and the all the electrons are attracted. The probe is in the electron collecting regime and there is no electron emission: this corresponds to the electron saturation current.

The third region $(\mathrm{BC})$ is the transition region between the electron emitting and electron collecting regime. In this region, the probe voltage lies near the plasma potential that one wants to measure. The probe current follows a exponential growth law characterized by the probe temperature $T_{w}$ as the emitted electrons are assumed to 
Evaluating the uncertainty for a complex experiment: the case of the plasma potential measurement 12

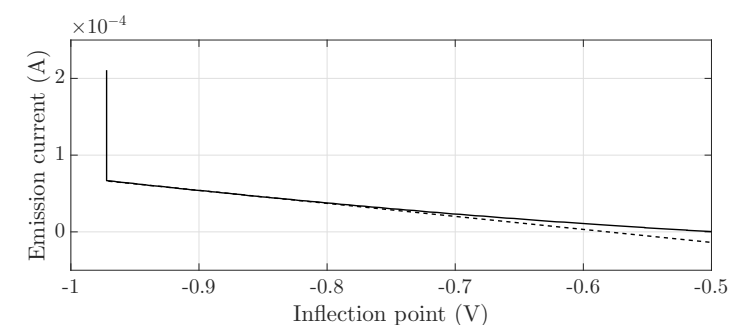

Figure 6. Theoretical relationship between the emission current and the inflection point (plain line). At very low emission the emission current varies non-linearly with the inflection point. At high emission it saturates (which is the regime used for an other measurement technique: the saturation method [5]). The dashed line corresponds to the linear fit used to extrapolate the plasma potential.

be described by a maxwellian distribution function at temperature $T_{w} \sharp$. The inflection point of this exponential region, which is the maximum of $\mathrm{d} I / \mathrm{d} V$, is the plasma potential [5]. However, because of the electron emission a negative space-charge forms near the probe and shifts the inflection point from the plasma potential toward lower values. It has been shown that in the limit of low electron emission, the shift grows linearly with the emssion current [11]. This linear growth can be experimentally measured to extrapolate the value of the inflection point at zero emission, which is the plasma potential (see Fig. 6). Thus this plasma potential measurement method is called the "inflection point in the limit of zero emission method".

It is within this framework that we propose a metrological procedure to determine the uncertainty of the plasma potential measurement. More precisely and in order to avoid any misunderstanding, in this work our goal is to provide a correct metrological procedure to determine the uncertainty measurement of a potential measured using the inflection point method. The correctness of the models underlying this method are not discussed here.

\subsection{Plasma discharge and experimental apparatus}

The experiments are performed in a device equivalent to the one described in Ref [19]. The discharge is produced in Argon gas at a $10^{-4}$ mbar, resulting in a plasma with a density of $10^{16} \mathrm{~m}^{-3}$. Here the anode (A) is the grounded vacuum vessel, and the cathode (K) consists of Tungsten wires heated to thermionic emission. The emitted electrons, accelerated by the potential bias, ionize the gas and produce the plasma.

The emissive probe (Figure 4) consists of a $\sim 3 \mathrm{~mm}$ long, $0.125 \mathrm{~mm}$ diameter hairpin shaped Tungsten wire [21]. The probe is designed to minimize the perturbations induced by its presence in the plasma, and an adjustable current is flowing in the wire in order to induce thermionic emission as low as possible. That current ohmically heats the probe and is created by a battery powered DC current supply, allowing steady electron

\# For a classical Langmuir probe, the probe current follows an exponential growth law characterized by the plasma electrons temperature. 
1

2

3

4

5

6

7

8

Evaluating the uncertainty for a complex experiment: the case of the plasma potential measurement13

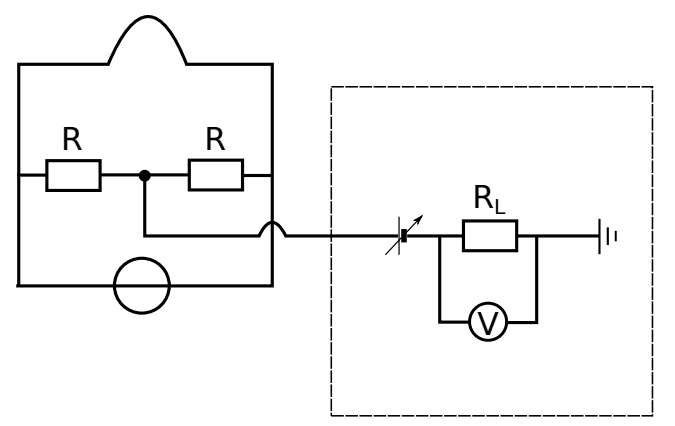

Figure 7. Heating and measuring probe circuit scheme. The probe is schemed by the circular arc. The dashed box represents the equivalent circuit of the probe driver.

emission, which is a mandatory feature for the measurements. The I-V characteristics of the probes are obtained thanks to a probe driver (ESPAS driver, designed and built at the University of Toulouse). The driver provides a -200 to $+100 \mathrm{~V}$ bias range using a 15 bits processor, giving a $0.0092 \mathrm{~V}$ resolution. The maximum current resolution is $1.5 \times 10^{-4} \mathrm{~mA}$. The emissive probe heating and measuring circuit is shown in Figure 7 : it consists of a battery powered DC current supply. Temperature variations may occur in time since the probe is gradually sputtered when negatively biased, leading to an increase of the resistance and consequently its temperature at constant heating current. However these variations occur on a large time scale (typically tens of minutes to hours) compared to the time needed to perform one characteristic (a few seconds). Smaller temperature variations may also happen, but they are highly unpredictable and random and therefore are part of the random error measurement. The measuring circuit is made of the two resistances $\mathrm{R}\left(100 \mathrm{k} \Omega\right.$ ) and the probe driver. The load $\mathrm{R}_{\mathrm{L}}$ fixes the current caliber and is tunable. The other driver components are not represented.

\section{Metrological analysis of the plasma potential measurement by an emissive probe}

\subsection{Description of the measuring chain}

The determination of the plasma potential is made as follows $\dagger \dagger$. After the discharge is switched on, the probe is heated to thermionic emission with the DC current supply. The typical bias ramp used to record the characteristics is $[-70,+20] \mathrm{V}$. The determination of the emission current requires sufficiently low biases in order to be far enough from the characteristic's inflection point (Fig 5), while the determination of the plasma potential requires enough data around its expected value (a few Volts in our device) to perform Savitzky-Golay filtering (a piece-wise polynomial fit of the experimental data) [22] that allows the calculation of the first derivative of the characteristic. The emission current available in specific toolboxes. It is however to perform this treatment with freewares and more common routines. 


\section{Evaluating the uncertainty for a complex experiment: the case of the plasma potential measurement14}

should be in the order of a few electron saturation current (part CD in Fig. 5), in order to stay in the low emission regime. Then, numerous characteristics are recorded, while varying the probe heating current. In order to avoid strong probe temperature variations during the measurements, they are made only after the stabilization of the heating current for the different setpoints.

For one given wire temperature $T_{w}$, one can measure the emission current $I_{e}$ and the potential at the infection point $V_{i}$ as follows. At each bias step the current is averaged over twenty samples, and the whole bias ramp is performed five times. This provides a better signal to noise ratio for the I-V characteristics. A calibration trace, which is an unloaded characteristic (i.e. the probe is unplugged), has to be subtracted from the experimental trace to take the electronic noise of the probe driver into account. First the characteristic is interpolated using piecewise cubic interpolation method (the number of points is conserved), and is then smoothed using a Stavitzky-Golay filter (a second order polynomial filter using a 21 points window). These parameters can of course be adapted depending on the signal-to-noise ratio of the characteristics. Since only the first derivative of the characteristic is computed, a second order polynomial is appropriate. The interpolation is necessary since the Savitzky-Golay filter shall be applied on an uniform grid [22]. Indeed, the experimental bias step provided by the probe driver fluctuates (it is a continuous random variable as well). The emission current $I_{e}$ is extracted from the mean of the current in the strongly negatively bias portion of the I- $\mathrm{V}$ characteristics, which is here taken as the first $10 \%$ of the characteristic $\dagger$. Finally it only remains to measure the potential at the inflection point $V_{i}$. The first derivative of the characteristics is computed using the differentiation matrix of the filter. Eventually, the maximum of the first derivative, i.e. the inflection point $V_{i}$, is recovered using a second order polynomial fit on the three points around the local maximum, which do not add any extra uncertainty (there is only one second order polynomial connecting three distinct points).

The $I_{e}=f\left(V_{i}\right)$ curve is plotted, and the linear region is identified by a clustering of the $I_{e}$ value. Clustering algorithms are made to find groups in data [23]. The algorithm used here is $k$-medoids based $\ddagger$. This method allows to group and discard data of the non-linear region, which are generally closer to each other than the points in the linear region (Figure 8). A linear fit is performed using a Monte Carlo algorithm $\S$ computing numerous linear least square regression on the data, in order to minimize the errors on the fit coefficients while taking into account the errors on $V_{i}$ and $I_{e}$. Finally the inflection point at zero emission, i.e. the plasma potential, and its absolute error (with

$\dagger$ Far enough means that $V_{i}-V_{\text {bias }} \ll e T_{e}$. This ensures that the probe is in the emission saturation regime.

$\ddagger$ It means that the algorithm attempts to group the data into $k$ different groups in which the distance between each point is minimal.

$\S$ A Monte Carlo algorithm relies on random sampling to perform a given task. Here the experimental points are randomly chosen in their probable range (given by their uncertainty) and the linear fit is performed, then this process is repeated. This allows the determination of the best fit and the standard deviation of its parameters. 
1

2

3

4

5

6

7

8

9

10

Evaluating the uncertainty for a complex experiment: the case of the plasma potential measurement 15
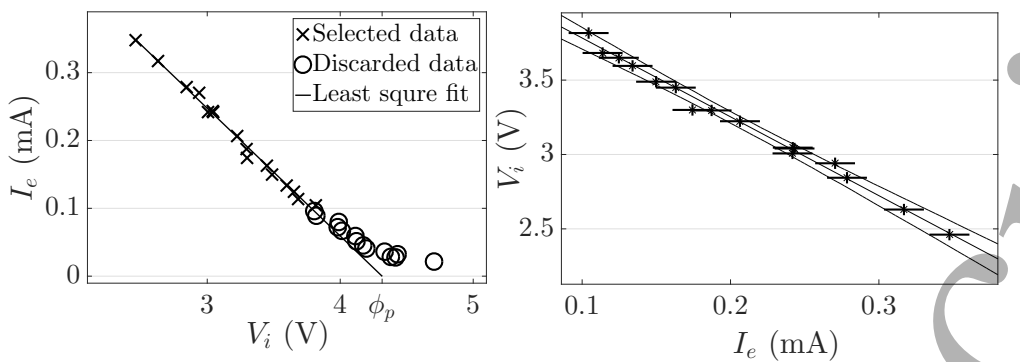

Figure 8. Data treatment for the linear fit. The non-linear part of the $I_{e}$ vs $V_{i}$ is discarded using a clustering algorithm (left figure). The least square regression is performed through a Monte-Carlo algorithm to find the best fit and minimize the uncertainty (right figure). The uncertainty of the fitting is represented by the dashed curves. The fit is extrapolated to $I_{e}=0$ to find the plasma potential $\phi_{p}$.

$95 \%$ confidence interval), are calculated from the linear fit $I_{e}=a V_{i}+b . \phi_{p}$ is the potential at which $I_{e}=0$, i.e. $\phi_{p}=-\frac{b}{a}$.

\subsection{Metrological procedure to determine the plasma potential uncertainty $u_{\phi_{p}}$}

Using the description of the measurement chain of the emissive probe diagnostic, the uncertainty of the plasma potential can be obtained by applying step by step the metrological procedure described in Sec.II.C.

\section{(i) Measurement modeling}

The plasma potential $\phi_{p}$ is determined indirectly by measuring $I_{e}$ (the emitted current)) and $V_{i}$ (potential at the inflection point). For $\mathrm{n}$ values of the probe temperature, n values of $I_{e}$ and $V_{i}$ are measured. $\phi_{p}$ is deduced from the linear regression curve $I_{e}=a V_{i}+b$. Moreover, a systematic shift is present in the model. Indeed, following [5], the inflection point method to determine $\phi_{p}$ with an emissive probe underestimates the plasma potential by a $\sim 0.1 k_{B} T_{e}[10]\left(k_{B}\right.$ is the Boltzmann constant and $T_{e}$ is the electron temperature). As a consequence, the measurement modeling leads to the following equation :

$$
\phi_{p}=-\frac{b}{a}+c=f(a, b, c),
$$

where the correction $c=0.1 k_{B} T_{e}$.

Applying the uncertainty propagation law, one can deduced the plasma potential uncertainty $u_{\phi_{p}}$ :

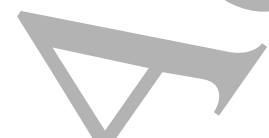

$$
u_{\phi_{p}}=\sqrt{\left(\frac{b}{a^{2}}\right)^{2} u_{a}^{2}+\left(\frac{-1}{a}\right)^{2} u_{b}^{2}+u_{c}^{2}},
$$

$u_{a}, u_{b}$ and $u_{c}$ being respectively the uncertainty of $a, b$ and $c$. 
Evaluating the uncertainty for a complex experiment: the case of the plasma potential measurement16

$u_{c}$ originates from the systematic shift of the measurement method discussed above and its value can be obtained by analyzing the origin of this error. This systematic error comes from the uncertainty of the measurement of the electron temperature $T_{e} \sim 2 \mathrm{eV}$. One can suppose that $T_{e}$ is a random variable following a normal law and, as a consequence, $u_{c}=\frac{0.1 k_{B} T_{e}}{3}$.

$u_{a}$ and $u_{b}$ come from the linear regression of the experimental curve $I_{e}=$ $a V_{i}+b$. More precisely, from the $n$ measurements of $I_{e}\left(I_{e_{1}}, I_{e_{1}}, \ldots, I_{e_{n}}\right)$ and $V_{i}$ $\left(V_{i_{1}}, V_{i_{2}}, \ldots, V_{i_{n}}\right)$, one can deduced $a$ and $b$ by a last squares method. Naturally, $u_{a}$ and $u_{b}$ are calculated from the uncertainties of the measured quantities, $i$. $e$. from $u_{I_{e}}$ and $u_{V_{i}}$. Supposing that i) $u_{I_{e}}<<u_{V_{i}}$ and ii) $u_{V_{i}}$ is constant for the $\mathrm{N}$ measurements, one can deduce $\|$

$$
\begin{aligned}
a & =\frac{\operatorname{cov}\left(I_{e} V_{i}\right)}{\sigma_{I_{e}}^{2}} \\
u_{a} & =\sqrt{\frac{u_{V_{i}}^{2}}{N \sigma_{I_{e}}^{2}}} \\
b & =\bar{V}_{i}-a \bar{I}_{e} \\
u_{b} & =\sqrt{\frac{u_{V_{i}}^{2}}{N}\left(1+\frac{\bar{I}_{e}^{2}}{\sigma_{I_{e}}^{2}}\right)}
\end{aligned}
$$

The hypothesis $\mathrm{i}$ and ii should be checked by calculating $u_{V_{i}}$ and $u_{I_{e}}$. If the hypothesis $\mathrm{i}$ is not satisfied and $u_{V_{i}} \ll u_{I_{e}}$, the measurement of $\phi_{p}$ has to be done using the linear regression curve $V_{i}=a^{\prime} I_{e}+b^{\prime}$ and following the same method.

(ii) Random error treatment

At this step, one has to calculate the random error on $I_{e}$ and $V_{i}$ for one probe temperature (i.e. for one given point of the regression curve). Five series of twenty characteristics with constant heating current were recorded in order to perform a $\chi^{2}$ test (with $5 \%$ significance level) on $V_{i}$ and $I_{e}$, which verifies that the sampling distribution may be considered as normal $₫$ [3]. Only twenty characteristics were measured for each set in order to minimize the influence of the slow temperature variation of the probe. Each set of twenty measurements validated the test. The mean sample standard deviations derived from these sets are $u_{I_{e}}^{\text {rand }}=0.0124 \mathrm{~mA}$ for $I_{e}$ and $u_{V_{i}}^{\text {rand }}=0.0311 \mathrm{~V}$ for $V_{i}$.

$\| \bar{I}_{e}=\sum_{j=1}^{n} I_{e_{j}} / n, \bar{V}_{i}=\sum_{j=1}^{n} V_{i_{j}} / n$ are respectively the experimental means of $I_{e}$ and $V_{i}$. $\sigma_{I_{e}}=\sqrt{\sum_{j=1}^{n}\left(I_{e_{j}}-\bar{I}_{e}\right)^{2} / n(n-1)}, \sigma_{V_{i}}=\sqrt{\sum_{j=1}^{n}\left(V_{i_{j}}-\bar{V}_{i}\right)^{2} / n(n-1)}$ are respectively the standard deviations of $I_{e}$ and $V_{i}$ obtained from the $n$ measurements. $\operatorname{cov}\left(I_{e}, V_{i}\right)=\frac{1}{n} \sum_{j=1}^{n}\left(I_{e_{j}}-\bar{I}_{e}\right)\left(V_{i_{j}}-\bar{V}_{i}\right)$ is the covariance of $I_{e}$ and $V_{i}$.

T A $\chi^{2}$ test may be applied on any sample distribution in order to determine if it corresponds to a given theoretical distribution. More precisely, it tests the null hypothesis on the sample distribution. More details are given in $\operatorname{Ref}[3]$ 
Evaluating the uncertainty for a complex experiment: the case of the plasma potential measurement 17

\section{(iii) Systematic errors treatment for the measurement of $I_{e}$}

At this step, one has to calculate the systematic errors and their uncertainty on $I_{e}$ for one temperature wire $(i$. e. for one given point of the regression curve) by analysis the measuring chain.

- Resolution and calibration of the drivers $u_{I_{e}}^{\text {res}}$ : The ESPAS constructor informations give $u_{I_{e}}^{r e s-E}=1.5 \times 10^{-4} \mathrm{~mA}$ and $u_{I_{e}}^{r e s-S}=0.1 \mu \mathrm{A}$.

- Subtraction of the calibration curve $u_{I_{e}}^{c a l}$ : The calibration curve of the ESPAS driver is subtracted from the experimental characteristic. The uncertainty of that systematic error is obtained by the mean standard deviation of the difference between the calibration trace and its fit.

- Savitzky-Golay filtering $u_{I_{e}}^{\text {gol }}$ : The uncertainty caused by the Savitzky-Golay filtering is calculated from the standard deviation of the difference between the characteristics before and after the filtering.

- Average of the first $10 \%$ of the characteristics $u_{I_{e}}^{a v}$ : Finally, the emission current $I_{e}$ is extracted from the mean of the current in the first $10 \%$ of the characteristics. The uncertainty due to this last average is simply the associated standard deviation.

(iv) Systematic errors treatment for the measurement of $V_{i}$

From the chain measuring analysis, one can determine the systematic errors and their uncertainty on $V_{i}$ for current emission $I_{e}$.

- Resolution and calibration of the drivers $u_{V_{i}}^{r e s}$ : The constructors informations give respectively $u_{V_{i}}^{r e s-E}=0.0092 \mathrm{~V}$ and $u_{V_{i}}^{r e s-S}=25 \mathrm{mV}$.

- Subtraction of the calibration curve (for the ESPAS driver only) $u_{V_{i}}^{c a l}$ : The calibration curve of the ESPAS driver is subtracted from the experimental trace. One can note that the calibration curve is fitted with a polynomial of order four. As a consequence, $u_{V_{i}}^{c a l}=\mathrm{d} V^{4}, \mathrm{~d} V$ being the bias step.

- Savitzky-Golay filtering $u_{V_{i}}^{\text {gol }}$ : The uncertainty caused by the Savitzky-Golay filtering is $\mathrm{d} V^{2}$ since the polynomial used is of order two. Then, to obtained a measure of $V_{i}$, the first derivative of the characteristics is computed from the polynomial given by the Savitzky-Golay filtering. The derivative is exact and this operation does not lead to an uncertainty.

- $3 r d$ order interpolation $u_{V_{i}}^{\text {inter }}$ : Finally, the inflection point $V_{i}$ is recovered using an interpolation of ordre 3 . As a consequence $u_{V_{i}}^{\text {inter }}=\mathrm{d} V^{3}$.

(v) Determination of $u_{I_{e}}$ from the propagation law

From the treatment of the random error and of the systematic errors on $I_{e}$ and using the uncertainty propagation law (Eq.9), one can deduce that the uncertainty measurement on $I_{e}$

$u_{I_{e}}=\left(u_{I_{e}}^{r a n d^{2}}+u_{I_{e}}^{r e s 2}+u_{I_{e}}^{c a l^{2}}+u_{I_{e}}^{g o l}{ }^{2}+u_{I_{e}}^{a v 2}\right)^{\frac{1}{2}}$.

(vi) Determination of $u_{V_{i}}$ from the propagation law 
Evaluating the uncertainty for a complex experiment: the case of the plasma potential measurement18

\begin{tabular}{|c|c|c|}
\hline Measurement modeling & Procedure & Potential determination \\
\hline Random error & $y=f\left(z_{1}, z_{2}, \ldots, z_{n}\right)$ & $\begin{array}{c}\phi_{p}=-\frac{b}{a}+c \\
\text { with } I_{e}=a V_{i}+b \\
\text { and } c=0.1 T_{e}\end{array}$ \\
\hline Systematic error & $u_{z_{j}}^{\text {rand }}=\sqrt{\sum_{i=1}^{n} \frac{\left(z_{j_{i}}-\bar{z}_{j}\right)^{2}}{n(n-1)}}$ & $u_{V_{i}}^{\text {rand }}$ and $u_{I_{e}}^{\text {rand }}$
\end{tabular}

Table 1. Summary of the uncertainty determination method applied to the plasma potential measurement.

From the treatment of the random error and of the systematic errors on $V_{i}$ and using the propagation law (Eq.9), one can deduce that the uncertainty measurement on $V_{i}$

$$
u_{V_{i}}=\left(u_{V_{i}}^{r a n d}+u_{V_{i}}^{r e s 2}+u_{V_{i}}^{c a l^{2}}+u_{V_{i}}^{g o l^{2}}+u_{V_{i}}^{i n t e r}{ }^{2}\right)^{\frac{1}{2}} .
$$

(vii) Determination of the expended measurement uncertainty on the potential plasma $u_{\phi_{p}}$

Finally after checking that the hypothesis $\mathrm{i}$ and ii have been checked, $i . \quad e$. $u_{I_{e}}<<u_{V_{i}}$ and $V_{i}$ is constant for the $n$ measurements, one can calculate the expended measurement uncertainty on the plasma potential $u_{\phi_{p}}$ from Eq.11. We suppose that the application conditions of the limit central theorem are filled and we enlarge the uncertainty with the coefficient $k=2$.

The summary of the method applied to the plasma potential determination is shown in Table 1.

\subsection{Experimental results}

Three plasma potential measurements were performed for several discharge parameters (Table 2) following the procedure previously presented. For every measurements, between 30 and 50 characteristics were recorded, depending on the $\left(I_{e}, V_{i}\right)$ distributions, which has to be large enough for a good identification of the linear region used for the determination of the plasma potential (Figure 6 and Figure 8). The errors $u_{I_{e}}$ and $u_{V_{i}}$ are calculated for each characteristic, as explained in section III-B. The results are 
1

2

3

4

5

6

7

8

10

Evaluating the uncertainty for a complex experiment: the case of the plasma potential measurement19

\begin{tabular}{cccc}
\hline \hline Measure & $\begin{array}{c}\text { Pressure } \\
\text { (mbar) }\end{array}$ & $\begin{array}{c}\text { Discharge } \\
\text { voltage }(\mathrm{V})\end{array}$ & $\begin{array}{c}\text { Discharge } \\
\text { current }(\mathrm{A})\end{array}$ \\
\hline 1 & $10^{-4}$ & 120 & 0.5 \\
2 & $10^{-4}$ & 120 & 0.25 \\
3 & $5 \times 10^{-5}$ & 120 & 0.5 \\
\hline \hline
\end{tabular}

Table 2. Plasma discharge parameters used for the plasma potential measurements.

\begin{tabular}{cccc}
\hline \hline Measure & $\begin{array}{c}\phi_{p} \\
(\mathrm{~V})\end{array}$ & $\begin{array}{c}\text { Absolute } \\
\text { uncert. (V) }\end{array}$ & $\begin{array}{c}\text { Relative } \\
\text { uncert.(\%) }\end{array}$ \\
\hline 1 & 4.32 & 0.09 & 2.0 \\
2 & 1.58 & 0.05 & 3.2 \\
3 & 0.42 & 0.02 & 3.4 \\
\hline \hline
\end{tabular}

Table 3. Results of the plasma potential measurements. The absolute errors are given with a $95 \%$ confidence interval.

Figure 9. Proportions of systematic and random errors on the overall errors on $V_{i}$ and $I_{e}$, for the measure number 1 .

presented in Table 3. The several contributions to the errors on $V_{i}$ and $I_{e}$ are shown in Figure 9.

The main contribution to the uncertainty on $V_{i}$ and $I_{e}$ is the random error, which was estimated by performing several measurements in the same experimental conditions. This suggests that the repeatability of the experiment is rather a strong hypothesis, and that some physical quantities, including the plasma potential itself, may encounter non negligible variations from one measurement to another. One can note that although the random error is the main contribution to the uncertainty, the systematic errors remain of the sâme order of magnitude. This shows that the standard deviation method is not sufficient to determine the measurement uncertainty.

The evolution of the plasma potential along the three measurements is beyond the scope of this paper. 
Evaluating the uncertainty for a complex experiment: the case of the plasma potential measurement20

\section{Conclusion}

The full method for the uncertainty determination was explained and given step-bystep. This method can be applied to any measurements, from simple ones to the most complex. It is important to understand that, in physics, a measured quantity never consists of a simple numerical value with an arbitrary precision. The probabilistic nature of the physical quantities leads only to the evaluation of the quantity and its uncertainty. This evaluation follows the step-by-step procedure. One can underly that the uncertainty evaluation method presented in this paper can be used to help students in physics (under graduated and graduated) to have a reflection on what a measurement in physics is.

This procedure was applied to the measurement of the plasma potential in a quiescent plasma discharge using an emissive probe. This kind of experiments may be performed by plasma physics graduate students, since the emissive probes constitute one of the basic plasma physics measurement tools. Here, the plasma potential is determined using the inflection point in the limit of zero emission method. This indirect measurement is a good example of a complex measurement, on which the step-by-step procedure mentioned before is rarely applied. It has been shown that it can indeed be done. The careful evaluation of the systematic errors and the random errors allows the rigorous calculation of the uncertainty. Moreover, this calculation also allows students to use the previous knowledge in data processing using numerical tools.

Numerous extensions of this work are possible. Especially, some of those may be performed and discussed by the students when they are performing the experiments, like: limiting situations where the measurements could not be performed; the influence of the variations of other parameters that would not have been taken into account in the present study; the replacement of one piece of equipment by another (especially the probe driver or the probe itself). It would be also interesting to apply the uncertainty determination to Langmuir probe measurements (ubiquitous in plasma physics experiments) and compare and discuss the possible differences with the emissive probe measurements. Finally, one can also discuss the limits of the models and assumptions that are used within these measurements: the assumed maxwellian nature of the electron distribution function is at the core of the theories of emissive probe characteristics analysis.

\section{References}

[1] International vocabulary of metrology - Basic and general concepts and associated terms (VIM), (JCGM 200, 2012)

[2] Evaluation of measurement data - Guide to the expression of uncertainty in measurement, (JCGM $1002008)$

[3] B. Grais, Méthodes Statistiques, Techniques statistiques, Tome 2, edited by Dunod (2003)

[4] I. Langmuir, J. Franklin Inst. 196 751-62 (1923)

[5] J. P. Sheehan and N. Hershkowitz, Plasm. Sour. Sci. Tech. 20, 063001 (2011)

[6] M. A. Lieberman and A. J. Lichtenberg, "Plasma Discharges and Materials Processing", John Wiley \& Sons, Inc. (2005) 
1

2

3

4

5

6

7

8

9

Evaluating the uncertainty for a complex experiment: the case of the plasma potential measurement21

[7] R. F. Kemp and J. M. Sellen Rev. Sci. Instrum. 37, 455 (1966)

[8] R. Schrittwieser et al., it Plasma Phys. Control. Fusion 44, 567 (2002)

[9] L. Oksuz and N. Hershkowitz, Plasma SOurces Sci. Technol. 14, 201 (2005)

[10] J. P. Sheehan, N. Hershkowitz, I. D. Kaganovich, H. Wang, Y. Raitses, E. V. Barnat, B. R. Weatherford and D. Sydorenko, Phys. Re. Lett. 111075002 (2013)

[11] M. Y. Ye and S. Takamura, Phys. Plasmas, 73457 (2000)

[12] J. P. Tierno et al., Phys. Plasmas 23, 013503 (2016)

[13] G. Bousselin el al., Phys. Plasmas 22, 053511 (2015)

[14] J. Cavalier, Phys. Plasmas 24, 013506 (2017)

[15] C. R. Hoffmann and D. J. Lees, Phys. Plasmas 13, 689 (1971)

[16] J. R. Smith, N.Hershkowitz and P. Coakley, Rev. Sci. Instrum. 50210 (1979)

[17] K. Ishikawa, Introduction to quality control, edited by Springer (1990)

[18] K. Protassov, "Analyse statistique des donnés expérimentales, Grenoble Sciences (2002)

[19] M. Carrère, L. Chérigier, C. Arnas-Capeau, G. Bachet and F.Doveil, Rev; Sci. Instrum. 674124 (1996)

[20] E. Stamate, K. Inagaki, K. Ohe and G.Popa, Journ. Phys. D 32671 (1999)

[21] A. Siebenförcher and R. Schrittwieser, Rev. Sci. Instrum. 67849 (1996)

[22] A. Savitzky and M. J. Golay, Anal. Chem. 361627 (1964)

[23] L. Kaufman and P. J. Rousseeuw, "Finding Groups in Data", John Wiley \& Sons, Inc. (1990)

[24] L. Oksuz and N. Hershkowitz, Phys. Letters A, 3752162 (2011) 